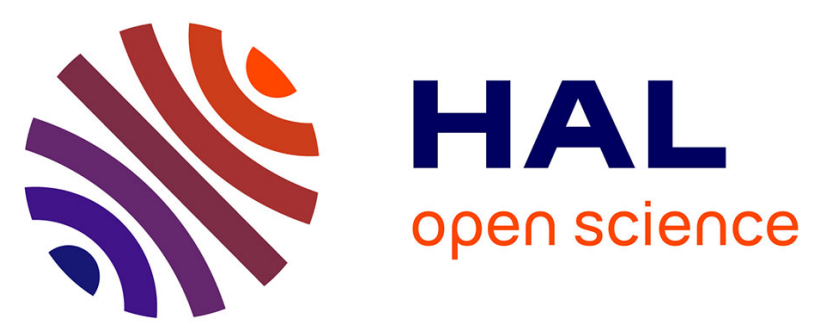

\title{
Complémentation d'un ensilage de ray-grass avec de l'orge, de la pulpe de betteraves ou du lactoserum: influence sur la digestibilité et sur la rétention azotée mesurées sur des moutons en croissance
}

Élisabeth Grenet, J. M. Boissau, H. Bousquet, Madeleine Dudilieu, Marie Jailler, Jacqueline Jamot, L. L'Hotelier

\section{To cite this version:}

Élisabeth Grenet, J. M. Boissau, H. Bousquet, Madeleine Dudilieu, Marie Jailler, et al.. Complémentation d'un ensilage de ray-grass avec de l'orge, de la pulpe de betteraves ou du lactoserum: influence sur la digestibilité et sur la rétention azotée mesurées sur des moutons en croissance. Annales de zootechnie, 1978, 27 (1), pp.115-122. hal-00887795

\section{HAL Id: hal-00887795 https://hal.science/hal-00887795}

Submitted on 1 Jan 1978

HAL is a multi-disciplinary open access archive for the deposit and dissemination of scientific research documents, whether they are published or not. The documents may come from teaching and research institutions in France or abroad, or from public or private research centers.
L'archive ouverte pluridisciplinaire HAL, est destinée au dépôt et à la diffusion de documents scientifiques de niveau recherche, publiés ou non, émanant des établissements d'enseignement et de recherche français ou étrangers, des laboratoires publics ou privés. 
Ann. Zootech., I978, 27 (I), II5-122.

Note

\title{
Complémentation d'un ensilage de ray-grass avec de l'orge, de la pulpe de betteraves ou du lactoserum : influence sur la digestibilité et sur la rétention azotée mesurées sur des moutons en croissance
}

\author{
Iisabeth GRENET \\ avec la collaboration technique de J. M. Boissat, H. Bousquer, \\ Madeleine DUdr,IEU, Marie JAIr,ER, Jacqueline JAmót et L. L'Hoté,IER \\ Laboratoire des Aliments, \\ Centre de Recherches de Clermont-Ferrand, I.N.R.A., \\ Theix, St-Genès-Champanelle, 63IIo Beaumont (France).
}

\section{Résumé}

A partir d'un ray-grass italien du I ${ }^{\text {er }}$ cycle au stade fin montaison que nous avons distribué à volonté en vert à un lot de 6 moutons sur lesquels nous avons mesuré la rétention azotée, nous avons préparé un ensilage en coupe directe et en brins courts, sans addition de conservateur. Cet ensilage a été distribué seul à 8 moutons âgés de 8 mois et pesant $40 \mathrm{~kg}$ environ à raison de $800 \mathrm{~g}$ de matière sèche par jour, ou complémenté avec de l'orge (310 $\mathrm{g}$ de matière sèche d'orge et $640 \mathrm{~g}$ de matière sèche d'ensilage), de la pulpe déshydratée de betteraves $\left(35^{\circ} \mathrm{g}\right.$ de pulpes et $600 \mathrm{~g}$ d'ensilage), ou du lactosérum sec (300 $\mathrm{g}$ de lactosérum et $650 \mathrm{~g}$ d'ensilage). Le dispositif expérimental était un carré latin avec 4 traitements et 2 moutons par traitement.

La digestibilité de la matière organique de l'ensilage distribué seul est identique à celle du vert mais la digestibilité des matières azotées et de la cellulose brute est plus élevée et la quantité ingérée est plus faible ainsi que la quantité d'azote retenue. Pour les rations d'ensilage complémentées la digestibilité de la matière organique, les quantités de matière sèche et de matière organique digestible ingérées sont plus élevées que pour l'ensilage seul, et la quantité d'azote retenue est améliorée sans qu'il y ait de différences significatives entre les compléments. Les résultats sont discutés en fonction de la composition du liquide du rumen et de la nature de chacun. des compléments. 


\section{Introduction}

La teneur en azote soluble des ensilages de fourrages verts, même bien conservés, est toujours supérieure à celle des fourrages verts ou des foins correspondants. Il en résulte d'une part une production accrue d'ammoniaque dans le rumen, et d'autre part une diminution de la quantité des protéines de ces ensilages qui atteint le duodénum après avoir échappé à la dégradation dans le rumen (BEEVER et al., I977). En même temps, la quantité d'énergie rapidement fermentescible des ensilages est plus faible que celle des fourrages verts, les glucides solubles ayant presque totalement disparu par suite des fermentations dans le silo. Un excès d'ammoniaque existe souvent dans le rumen d'où une excrétion d'azote urinaire accrue et une diminution de la quantité d'acides aminés mise à la disposition de l'animal, donc de la quantité d'azote retenue. L'apport d'un complément, riche en énergie digestible, avec les ensilages de fourrages verts pouvant améliorer la quantité d'azote retenue (Conrad et al., I96r; Ferrando et Catsaounis, i966; Durand, Zelter et Tisserand, ig68; 'Thomson, i968; Griffiths, SpilIANE et BATH, I973) nous avons comparé trois sources d'énergie : de l'orge, de la pulpe de betteraves et du lactosérum, pour étudier si, à même quantité d'énergie apportée, elles étaient ou non équivalentes entre elles pour améliorer l'utilisation de l'azote des ensilages d'herbe et quelle était l'importance de cette amélioration.

\section{Matériel et méthodes}

Le fourrage utilisé est un premier cycle de ray-grass italien au stade fin montaison contenant $\mathrm{I} 4 \mathrm{p}$. cent de matière sèche et dont la teneur en matières azotées est de 13,9 p. cent de la matière sèche. Nous avons distribué ce fourrage en vert, à volonté, à un lot de 6 moutons âgés de 15 mois et pesant $47 \mathrm{~kg}$ en moyenne, et sur lesquels nous avons mesuré la rétention azotée (GRENET et DEMarQUILIY, 1977). Nous avons préparé un ensilage avec ce fourrage, en coupe directe et en brins courts, mais sans addition de conservateur, dans de petits silos de $5 \mathrm{~m}^{3}$ en butyl. Sa teneur en matière sèche est de $\mathrm{I} 5$, I p. cent, sa teneur en matières azotées de 14,7 p. cent de la matière sèche et son $\mathrm{pH}$ de 4,53; ses teneurs en azote ammoniacal et en azote soluble sont respectivement de I0,4 et de 50,5 p. cent de l'azote total. Sa teneur en acide acétique est très élevée $(87 \mathrm{~g} / \mathrm{kg}$ de matière sèche) mais celle en acide butyrique est très faible $(0,5 \mathrm{~g})$.

Cet ensilage a été distribué à 8 moutons mâles castrés de race Texel, âgés de 8 mois et pesant $40 \mathrm{~kg}$ environ, soit seul, en quantité limitée à $90 \mathrm{p}$. cent de la quantité ingérée ad libitum, c'est-à-dire $800 \mathrm{~g}$ de matière sèche par jour, soit complémenté avec :

de l'orge (I5,8 de matières azotées $\mathrm{p}$. cent de la matière sèche) dans la proportion de $33 \mathrm{p}$. cent d'orge et de $67 \mathrm{p}$. cent d'ensilage, c'est-à-dire $310 \mathrm{~g}$ de matière sèche d'orge et $640 \mathrm{~g}$ de matière sèche d'ensilage, car nous avons considéré que la distribution de 3 Io $\mathrm{g}$ d'orge ferait baisser la consommation d'ensilage de I60 $\mathrm{g}$ environ;

de la pulpe déshydratée de betteraves (Io,o de matières azotées p. cent de la matière sèche) à raison de $350 \mathrm{~g}$ de pulpes et de $600 \mathrm{~g}$ d'ensilage; 
ou du lactosérum sec ( 13,8 de matières azotées p. cent de la matière sèche) à raison de $300 \mathrm{~g}$ de lactosérum et de $65^{\circ} \mathrm{g}$ d'ensilage.

Les quantités de pulpes et de lactosérum distribuées ont été calculées de telle sorte qu'elles apportent la même quantité d'énergie que les 3 Io g d'orge. Le complément était distribué en 2 repas égaux avant chaque distribution d'ensilage.

Le dispositif expérimental adopté était un carré latin avec 4 traitements et 2 moutons par traitement, les périodes pré-expérimentales étant de I I jours et les périodes de mesure de ro jours. La mesure de la rétention azotée et les prélèvements de jus de rumen ont été réalisés avec les mêmes méthodes que celles utilisées pour les fourrages verts (GRENET et I)EMARQUILI,Y, I977).

\section{Résultats et discussion}

La digestibilité de la matière organique de l'ensilage distribué seul est identique à celle du fourrage vert mais la digestibilité des matières azotées et de la cellulose brute est plus élevée et la quantité ingérée plus faible (tabl. r). La quantité d'azote retenue est nettement plus faible avec l'ensilage : 3,4 contre $6,2 \mathrm{~g}$ par jour avec le fourrage vert (tabl. 2), mais les quantités d'azote et de matière organique diges-

TABLEAU I

Digestibilité et quantités ingérées

Digestibility and voluntary intake

\begin{tabular}{|c|c|c|c|c|c|}
\hline \multirow{2}{*}{$\begin{array}{l}\text { Régine } \\
\text { (Diet) }\end{array}$} & \multicolumn{3}{|c|}{$\begin{array}{l}\text { Coefficient de digestibilité p. cent } \\
\text { (Digestibility p. cent) }\end{array}$} & \multirow{2}{*}{$\begin{array}{c}\text { Quantité } \\
\text { de matière } \\
\text { sèche ingérée } \\
\text { (g/kg P.0.75) } \\
(\text { Iry matter } \\
\text { intake } \\
\left.\left(g, k g P^{2}, 7^{2}\right)\right)\end{array}$} & \multirow{2}{*}{$\begin{array}{l}\text { Quantité } \\
\text { de matière } \\
\text { organique } \\
\text { digestible } \\
\text { ingérée }(\mathrm{g} / \mathrm{j}) \\
(\text { Digestible } \\
\text { organic matter } \\
\text { intake }(g / d))\end{array}$} \\
\hline & $\begin{array}{l}\text { Matière } \\
\text { organique } \\
\text { (Organic } \\
\text { matter) }\end{array}$ & $\begin{array}{l}\text { Matière } \\
\text { azotée } \\
\text { (Crude } \\
\text { protein) }\end{array}$ & $\begin{array}{l}\text { Cellulose } \\
\text { brute } \\
\text { (Crude } \\
\text { fiber) }\end{array}$ & & \\
\hline Herbe (Fresh grass). & 74,4 & 63,8 & 69,0 & 73,2 & 857 \\
\hline 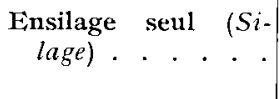 & $74,7^{a} \underset{(\mathbf{r})}{0,7}$ & 68,0 & 78,8 & $53,2^{a} \pm 3,2$ & $600^{a} \pm 39$ \\
\hline $\begin{array}{l}\text { Ensilage }+ \text { orge (Si- } \\
\text { lage }+ \text { barley) } \\
\text { Ensilage }+ \text { pulpe }\end{array}$ & $77,1^{\nu} \pm 0,8$ & 70,1 & 73,9 & $61,3^{b} \pm 3,1$ & $754^{b}=33$ \\
\hline $\begin{array}{l}\text { (Silage +beet } \\
\text { pulp). } \\
\text { Fnsilage + lactosé- } \\
\text { rum (Silage + } \\
\text { whey).... }\end{array}$ & $\begin{array}{l}77,5^{\circ} \pm 1,2 \\
77,8^{b} \pm 0,9\end{array}$ & 63,5 & 79,2 & $60,2^{b} \pm 5,5$ & $\begin{array}{l}764^{b}=52 \\
74^{8 b} \pm 22\end{array}$ \\
\hline
\end{tabular}

(') Les valeurs suivies de la mème lettre ne isont pas significativement différentes entre elles $(\mathbf{P} \leqslant 0,05)$. Values followed by the same letter are not significantly different $(P \leqslant 0,05)$. 


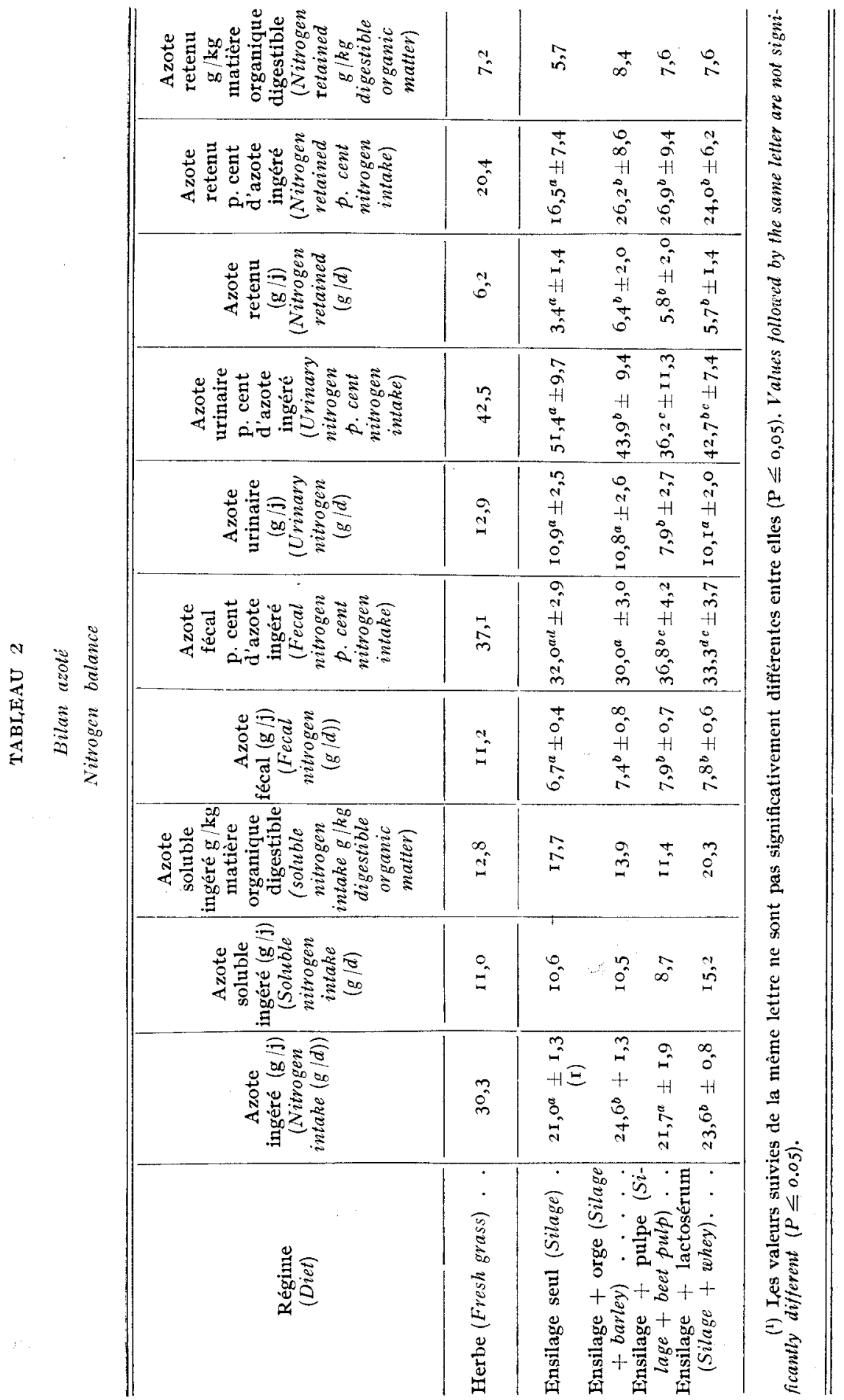


tible ingérées sont bien plus faibles pour l'ensilage (respectivement $2 \mathrm{I}$ et $600 \mathrm{~g} / \mathrm{j}$ ) que pour le fourrage vert $(30,3$ et $857 \mathrm{~g} / \mathrm{j})$.

La digestibilité de la matière organique, la quantité de matière sèche ingérée et la quantité de matière organique digestible ingérée sont significativement plus élevées pour les rations d'ensilage complémentées que pour l'ensilage seul, mais elles ne sont pas significativement différentes entre elles selon la nature du complément. Comme cela pouvait être prévu d'après le protocole expérimental, la quantité d'azote ingérée est plus élevée pour les rations complémentées avec de l'orge ou du lactosérum que pour l'ensilage seul ou l'ensilage avec de la pulpe. La quantité d'azote retenue, en g par jour ou en p. cent de l'azote ingéré, est améliorée par la complémentation énergétique (tabl. 2) sans qu'il y ait de différences significatives entre orge, pulpe ou lactosérum. L'augmentation de la quantité d'énergie apportée aux microorganismes du rumen a permis une synthèse microbienne plus importante; il en résulte une diminution de la teneur en azote ammoniacal du jus de rumen dont la valeur moyenne par jour passe de $254 \mathrm{mg} / 1$ à I5 I mg/1 en moyenne, donc des pertes d'ammoniaque au niveau du rumen et des pertes d'azote dans l'urine, et une augmentation de la quantité d'azote protéique arrivant dansle duodénum, qui expliquent l'augmentation de la quantité d'azote retenue. Si on exprime cette dernière en fonction de la quantité de matière organique digestible ingérée, elle est nettement augmentée par la complémentation passant de $5.7 \mathrm{~g}$ d'azote retenu par $\mathrm{kg}$ de matière organique digestible ingérée pour l'ensilage seul à $8 \mathrm{~g}$ pour les ensilages complémentés.

On ne note cependant pas d'augmentation de la concentration en acides gras volatils du liquide du rumen avec les rations complémentées, mais une orientation différente des fermentations : elles conduisent à la formation de davantage d'acides propionique et butyrique aux dépens de l'acide acétique (tabl. 3 ). La concentration en acide acétique est très élevée en moyenne, probablement parce que l'ensilage en renferme beaucoup.

La proportion d'azote excrétée dans les fèces est significativement plus élevée pour l'ensilage avec pulpe sans doute par suite de la présence d'énergie fermentescible dans le gros intestin (ThORNTON et YATES, I968), que pour les autres rations, tandis que la ration d'ensilage avec orge d'une part, et celle d'ensilage avec lactosérum d'autre part, ne diffèrent pas significativement de la ration d'ensilage seul. La teneur en azote soluble des pulpes est faible, et la quantité d'azote soluble ingérée en $\mathrm{g}$ par $\mathrm{kg}$ de matière organique digestible est de I I,4 seulement pour la pulpe contre I3,9 pour l'ensilage avec orge et 20,3 pour l'ensilage avec lactosérum. Ceci explique la faible teneur en azote ammoniacal du jus de rumen (tabl. 3) et la faible excrétion d'azote dans l'urine avec la pulpe (tabl. 2 ). Cependant si on admet que 35 p. cent de l'azote insoluble ingéré sont aussi transformés en $\left(\mathrm{NH}_{3}\right)$ dans le rumen, la quantité de $\mathrm{N} \times 6,25$ fermentescible ingéré par $\mathrm{kg}$ (MOD) est de Io $8 \mathrm{~g}$ alors que la possibilité de synthèse d'azote microbien est de l'ordre de $135 \mathrm{~g} \mathrm{~N} \times 6,25 / \mathrm{kg}$ MOD. La teneur en $\mathrm{NH}_{3}$ du jus de rumen aurait donc dû être pratiquement nulle en l'absence d'un recyclage important d'azote avec ce régime. En revanche la teneur en ammoniaque du liquide du rumen et l'excrétion d'azote urinaire sont du même ordre de grandeur pour l'orge et le lactosérum, bien que les matières azotées du lactosérum soient entièrement solubles et que celles de l'orge ne le soient qu'à 25 p. cent. Il faut donc admettre que malgré ces différences de solubilité, la fermentescibilité (transformation en ammoniaque dans le rumen) des matières azotées de ces deux aliments est voisine, ou alors qu'à même quantité d'énergie apportée, le lactose permet une synthèse microbienne plus intense que l'amidon de l'orge. La concentration en acides gras 


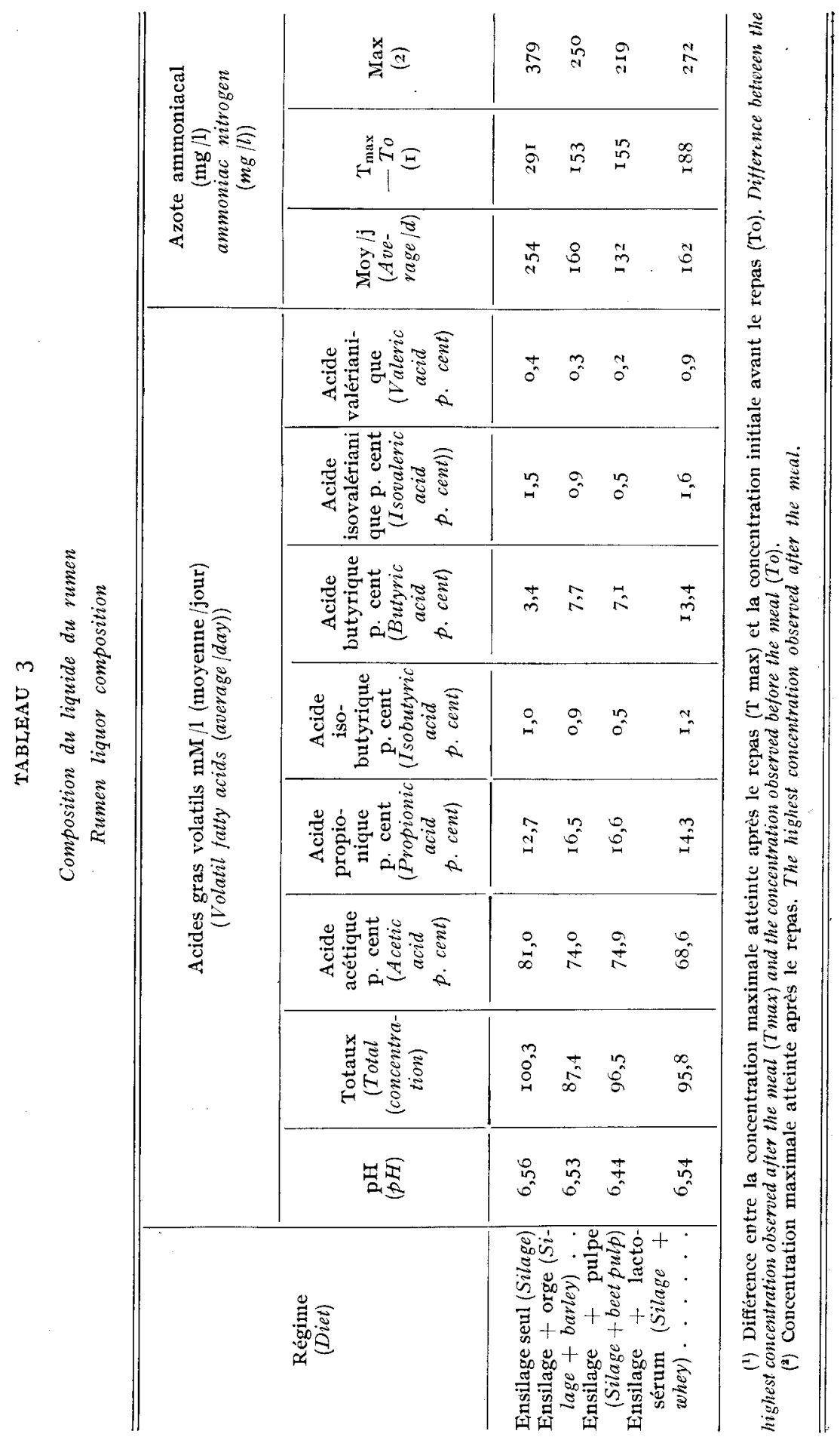


volatils du liquide du rumen montre une augmentation des acides butyrique et valérianique avec le lactosérum par rapport aux ensilages avec l'orge ou la pulpe, et une diminution corrélative des acides acétique et propionique comme l'ont montré DE Vuyst et al., I970, ce qui indique une orientation de la flore du rumen différente avec ce régime.

Les résultats obtenus montrent que la complémentation énergétique des ensilages d'herbe permet d'améliorer sensiblement la rétention azotée par des moutons en croissance, contrairement à ce que nous avions observé avec des fourrages verts (GRENET et DEMARQUII, Y, I977). Pourtant la dégradation des matières. azotées entraînées par les processus de récolte et d'ensilage n'était pas très importante dans cet essai, la moitié seulement de l'azote de l'ensilage étant sous forme soluble. Cette amélioration de la rétention azotée est en outre indépendante de la nature des glucides ajoutés : amidon, substances pectiques très digestibles ou lactose.

$$
\text { Accepté pour publication en octobre } 1977 \text {. }
$$

\section{Summary}

\section{Supplementation of rye-grass silage with barley, sugar beet pulp} or whey: infuence on digestibility and nitrogen retention measured in growing sheep.

First cut Italian rye-grass at the stage of late stem elongation was offered directly ad libitum to a group of 6 sheep in which the nitrogen retention was measured. A silage was prepared from this rye-grass (direct cut, finely chopped) without any additive. This silage was given to 8 growing sheep aged 8 months and weighing about $40 \mathrm{~kg}$, at the rate of $800 \mathrm{~g}$ dry matter per day or supplemented with barley (3 $10 \mathrm{~g}$ barley dry matter and $640 \mathrm{~g}$ silage dry matter), dried sugar beet pulp (350 $\mathrm{g}$ pulp and $600 \mathrm{~g}$ silage) or dry whey (300 $\mathrm{g}$ whey and $650 \mathrm{~g}$ silage). The experimental design was a latin square with 4 treatments and 2 sheep per treatment.

The digestibility of organic matter of the silage offered alone was the same as that of fresh rye-grass while the digestibility of crude protein and crude fiber was higher and the voluntary intake as well as the amount of nitrogen retained was lower. For the supplemented silage diets, the digestibility of organic matter as well as the intake of dry matter and digestible organic matter were higher than for silage alone. All supplements significantly improved nitrogen retention. but differences between supplements were not significant. Results are discusssed in relation to the composition of rumen liquor and the nature of each supplement.

\section{Références bibliographiques}

BFever D. E., 'Thomson D. J., Cammer S. B. et Harrison D. G., I977. 'The digestion by sheep' of silages made with and without the addition of formaldehyde. $J$. A gric. Sci., 88, 6r-70.

Conrad H. R., Hibbs J. W., Prat'T A. D, et Davis R. R., r96r. Nitrogen metabolism in dairy cattle. I - The influence of grain and meadow crops harvested as hay, silage, or soilage on efficiency of nitrogen utilization. $J$. Dairy Sci., 44, 85-95.

De Vuyst A., Jaramillo D., Vanbelif M., Arnould R. et Crevecaeur E., ig7o. Influence de l'addition de saccharose et de lactose à la ration du mouton sur la composition du contenu. du rumen. Publication du Centre de Recherches Zootechniques de l'Université de Louvain, 4, I-23.

DURAND M., ZEITER S. Z. et TISSERAND J. L., I968. Influence de quelques techniques de conservation sur l'efficacité de l'azote de la luzerne chez le mouton. Ann. Biol. anim. Bioch. Biophys, 8, $45-67$.

Ffrrando R. et Catsaounis N., i966. Signification de la digestibilité des matières azotées. chez les ruminants. Ann. Nutr. Al., 20, 127-145. 
GRFNET E. et DEMARQUir., C., I977. Utilisation de l'azote des fourrages, verts par le mouton en croissance : influence du stade de végétation, de l'espèce fourragère, de la fertilisation azotée et de l'addition d'orge. Ann. Zootech., 26, 48r-5or.

Griffiths T. W., Spillane T. A. et BATHS I. H., I973. Studies on the nutritive value of silage with particular reference to the effects of energy and nitrogen supplementation in growing heifers. $J$. agric. Sci, $80,75^{-88}$.

Thomson D. J., I968. The digestibility and utilization of fresh grass, Anim. Prod., 10, 240 (Abstr.).

Thornton R. F. et YATES N. G., I968. Some effects of water restriction on apparent digestibility and water excretion of cattle. Austr. J. Agric. Res., 19, 665-672. 